\title{
410.
}

\section{A THIRD MEMOIR ON SKEW SURFACES, OTHERWISE SCROLLS.}

[From the Philosophical Transactions of the Royal Society of London, vol. CLIx. (for the year 1869), pp. 111-126. Received May 30,-Read June 18, 1868.]

THE present Memoir is supplementary to my "Second Memoir on Skew Surfaces, otherwise Scrolls," Phil. Trans. vol. CLIv. (1864), pp. 559-577, [340], and relates also to the theory of skew surfaces of the fourth order, or quartic scrolls. It was pointed out to me by Herr Schwarz $\left(^{1}\right)$, in a letter dated Halle, June 1, 1867, that in the enumeration contained in my Second Memoir I heve given only a particular case of the quartic scrolls which have a directrix skew cubic; viz. my eighth species, $S\left(1,3^{2}\right)$, where there is also a directrix line. And this led me to observe that I had in like manner mentioned only a particular case of the quartic scrolls with a triple directrix line; viz. my third species, $S\left(1_{3}, 1,4\right)$, where there is also a simple directrix line. The omitted species, say, ninth species, $S\left(1_{3}\right)$, with a triple directrix line, and tenth species, $S\left(3^{2}\right)$, with a directrix skew cubic, are considered in the present Memoir; and in reference to them I develope a theory of the reciprocal relations of these scrolls, which has some very interesting analytical features.

The paragraphs of the present Memoir are numbered consecutively with those of my Second Memoir above referred to.

\section{Quartic Scroll, Ninth Species, $S\left(1_{3}\right)$, with a triple directrix line.}

54. Consider a line the intersection of two planes, and let the equation of the one plane contain in the order 3 , that of the second plane contain linearly, a variable parameter $\theta$; the equations of the two planes may be taken to be

$$
(p, q, r, s \gamma \theta, 1)^{3}=0, \quad(u, v \gamma(\theta, 1)=0,
$$

${ }^{1}$ I take the opportunity of referring to his paper on Quintic Scrolls, Schwarz, "Ueber die geradlinigen Flächen fünften Grades," Crelle, t. Lxvir. (1867), pp. 23-57. 
where $(p, q, r, s, u, v)$ are any linear functions whatever of the coordinates $(x, y, z, w)$. Hence eliminating $\theta$ we have as the equation of the scroll generated by the line in question

$$
\left(p, q, r, s \gamma(v,-u)^{3}=0,\right.
$$

viz. this is a quartic scroll having the line $u=0, v=0$ for a triple line; that is, the line in question is a triple directrix line.

55. Taking $x=0, y=0$ for the equations of the directrix line, or writing $u=x$, $v=y$, and moreover expressing $(p, q, r, s)$ as linear functions of the coordinates $(x, y, z, w)$, the equation of the scroll takes the form

$$
(* \chi \chi x, y)^{4}+z(* \chi \chi x, y)^{3}+w\left(*^{\prime} \backslash(x, y)^{3}=0 ;\right.
$$

and we may, by changing the values of $z$ and $w$, make the term in $(x, y)^{4}$ to be

$$
\left(* \chi(x, y)^{4}+(\alpha x+\beta y)(* \chi \chi x, y)^{3}+(\gamma x+\delta y)\left(*^{\prime} \chi(x, y)^{3},\right.\right.
$$

where the arbitrary constants $\alpha, \beta, \gamma, \delta$ may be so determined as to reduce this to a monomial $k x^{4}, k x^{3} y$, or $k x^{2} y^{2}$.

56. The coefficient $k$ may vanish, and the equation of the scroll then is

$$
z(* \chi x, y)^{3}+w\left(*^{\prime} \chi(x, y)^{3}=0,\right.
$$

or, what is the same thing, it is

$$
(* \chi x, y)^{3}(z, w)=0 \text {, }
$$

viz. the scroll has in this particular case the simple directrix line $z=0, w=0$, thus reducing itself to the third species, $S\left(1_{3}, 1,4\right)$, with a triple directrix line and a single directrix line. It is proper to exclude this, and consider the ninth species, $S\left(1_{3}\right)$, as having a triple directrix line, but no simple directrix line.

57. The scroll $S\left(1_{3}\right)$ may be considered as a scroll $S(m, n, p)$ generated by a line which meets each of three given directrices; viz. these may be taken to be the directrix line, and any two plane sections of the scroll. The section by any plane is a quartic curve having a triple point at the intersection with the directrix line; moreover the sections by any two planes meet in four points, the intersections of the scroll by the line of intersection of the two planes. Conversely, taking any line and two quartics related as above (that is, each quartic has a triple point at its intersection with the line, and the two quartics meet in four points lying in a line), the lines which meet the three curves generate a quartic scroll $S\left(1_{3}\right)$. This appears from the formula

$$
S(m, n, p)=2 m n p-\alpha m-\beta n-\gamma p(\text { Second Memoir, No. 5); }
$$

we have in the present case

$$
m=1, n=4, p=4, \quad \alpha=4, \beta=3, \gamma=3,
$$

and the order of the scroll is $32-4-12-12,=4$, that is, the scroll is a quartic scroll; there is no difficulty in seeing that through each point of the line there pass c. VI. 
three generating lines, but through each point of either of the plane quartics only a single generating line; that is, that the line is a triple directrix line, but each of the plane quartics a simple directrix curve.

58. We may instead of the section by any plane, consider the section by a plane through a generating line, or by a plane through two of the three generating lines which meet at any point of the directrix line; if (to consider only the most simple case) each of the planes be thus a plane through two generating lines, the section by either of these planes is made up of the two generating lines, and of a conic passing through the directrix line; the directrices are thus the line and two conics each of them meeting the line; we have therefore in the foregoing formula

$$
m=1, n=2, p=2, \quad \alpha=0, \beta=1, \gamma=1,
$$

and the order of the scroll is $8-2-2,=4$ as before.

Quartic Scroll, Tenth Species, $\left(3^{2}\right)$, with a directrix skew cubic met twice by each generating line $\left.{ }^{1}\right)$.

59. Consider a line, the intersection of two planes; and let the equation of each plane contain in the order 2 a variable parameter $\theta$; the equations of the two planes may be taken to be

$$
(p, q, r \gamma \theta, 1)^{2}=0, \quad\left(p^{\prime}, q^{\prime}, r^{\prime} \gamma \theta, 1\right)^{2}=0,
$$

where $\left(p, q, r, p^{\prime}, q^{\prime}, r^{\prime}\right)$ are linear functions of the coordinates $(x, y, z, w)$; hence eliminating $\theta$, we have as the equation of the scroll generated by the line in question, $\square=0$, where $\square$ is the resultant of the two quadric functions. The equation may be written

$$
4\left(p q^{\prime}-p^{\prime} q\right)\left(r q^{\prime}-r^{\prime} q\right)-\left(p r^{\prime}-p^{\prime} r\right)^{2}=0 ;
$$

and the scroll has thus as a nodal (double) line the skew cubic determined by the equations

$$
\left\|\begin{array}{lll}
p, & q, & r \\
p^{\prime}, & q^{\prime}, & r^{\prime}
\end{array}\right\|=0
$$

It is easy to see (and indeed it will be shown presently) that this curve is met twice by each generating line of the scroll, and that the scroll is consequently a quartic scroll as described above.

${ }^{1}$ I have worded this heading in accordance with that of the eighth species, Second Memoir, No. 47, but the two headings might be expressed more completely thus :

Eighth Species, $S\left(1,3_{2}{ }^{2}\right)$, with a directrix line and a double directrix skew cubic met twice by each generating line;

Tenth Species, $S\left(3_{2}{ }^{2}\right)$, with a double directrix skew cubic met twice by each generating line;

viz. the subscript 2 would indicate that the skew cubic is a nodal (double) line on the scroll, the exponent 2 indicating that it is met twice by each generating line. 
60. The coordinates $(x, y, z, w)$ may be fixed in such manner that the equations of the skew cubic shall be

or, what is the same thing,

$$
\left\|\begin{array}{lll}
x, & y, & z \\
y, & z, & w
\end{array}\right\|=0
$$

$$
y w-z^{2}=0, \quad z y-x w=0, \quad x z-y^{2}=0
$$

each of the equations $p q^{\prime}-p^{\prime} q=0, r q^{\prime}-r^{\prime} q=0, p r^{\prime}-p^{\prime} r=0$ is then the equation of a quadric surface passing through the skew cubic, or, what is the same thing, each of the functions $p q^{\prime}-p^{\prime} q, r q^{\prime}-r^{\prime} q, p r^{\prime}-p^{\prime} r$ is a linear function of $y w-z^{2}, z y-x w, x z-y^{2}$; and the equation of the scroll is given as a quadric equation in the last-mentioned quantities. It will be convenient to represent the equation in the form

$$
\left(H, F, C, B, A-F,-G \gamma y w-z^{2}, z y-x w, x z-y^{2}\right)^{2}=0,
$$

or, writing for shortness

$$
y w-z^{2}, z y-x w, x z-y^{2}=p, q, r,
$$

which letters $(p, q, r)$ are used henceforward in this signification only, the equation will be

$$
\left(H, F, C, B, A-F,-G^{\gamma} \gamma p, q, r\right)^{2}=0,
$$

viz. this is a quadric equation in $(p, q, r)$, with arbitrary coefficients.

61. Comparing with the result, Second Memoir, Nos. 47 to 50, we see that in the particular case where the coefficients $(A, B, C, F, G, H)$ satisfy the relation $A F+B G+C H=0$, we have the eighth species, $S\left(1,3^{2}\right)$, with a directrix line and a directrix skew cubic met twice by each generating line. We exclude this particular case, and in the tenth species consider the relation $A F+B G+C H=0$ as not satisfied, and therefore the scroll as not having a directrix line.

62. I consider how the scroll may be obtained as a scroll $S\left(m^{2}, n\right)$ generated by a line meeting a curve of the order $m$ twice and a curve of the order $n$ once. The first curve will be the skew cubic, that is $m=3$; the second curve may be any plane section of the scroll; such a section will be a quartic curve having three nodes, one at each intersection of its plane with the skew cubic. Conversely, if we have a skew cubic, and a plane quartic meeting the skew cubic in three points, each of them a node on the quartic, then the scroll generated by the lines which meet the skew cubic twice and the quartic once will be a quartic scroll. In fact (see First Memoir, No. 10, [339], and Second Memoir, No. 5) the order of the scroll is given by the formula $S\left(m^{2}, n\right)=n\left([m]^{2}+M\right)$ - reduction, $=16$ - reduction. And in the present case the reduction arises (Second Memoir, No. 4) from the cones having their vertices at the intersections of the skew cubic and the quartic, and passing through the skew cubic. Each cone is of the order 2, and each intersection qua double point on the quartic gives a reduction $2 \times$ order of cone, $=4$; that is, the reduction arising from the three intersections is $=12$; or the order of the scroll is $16-12$, $=4$. 
63. We may, instead of the section by a plane in general, consider the section by a plane through a generating line; the section is here made up of the generating line and of a plane cubic passing through each of the two points of intersection of the generating line with the skew cubic, and having a node at the remaining intersection of its plane with the skew cubic. Or we may consider the section by a plane through the two generating lines at any point of the skew cubic; the section is here made up of the two generating lines and of a conic passing through the second intersections of the two generating lines with the skew cubic; that is, meeting the skew cubic twice.

64. Conversely, consider a skew cubic, and a conic meeting it twice; the lines which meet the skew cubic twice, and also the conic, generate a quartic scroll; this appears by the before-mentioned formula $S\left(m^{2}, n\right)=n\left([m]^{2}+M\right)$-reduction; viz. we have $m=3, n=2$, and the order is $=8$-reduction; the reduction arises from the cones having their vertices at the intersections of the skew cubic and the conic. Each cone is of the order 2, and (quà simple point on the conic) each intersection gives a reduction $=$ order of the cone; that is, the total reduction is $=4$, and the order of the scroll is $8-4,=4$ as above.

65. But a more elegant mode of generation of the scroll may be obtained by means of the skew cubic alone; viz. considering the system of lines which are in involution with five given lines, or say simply the lines which belong to an involution $\left({ }^{1}\right)$, I say that the locus of a line belonging to the involution, and meeting the skew cubic twice is the quartic scroll, tenth species, $S\left(3^{2}\right)$. In the particular case where the line (instead of belonging to a proper involution) meets a given line, the locus is a quartic scroll, eighth species, $S\left(1,3^{2}\right)$.

66. The analysis is almost identical with that given (Second Memoir, Nos. 47 to 50) in regard to the scroll $S\left(1,3^{2}\right)$. Considering a line defined by its "six coordinates" $(a, b, c, f, g, h)$, the condition which expresses that the line shall belong to an involution is

$$
(A, B, C, F, G, H \gamma a, b, c, f, g, h)=0,
$$

where $(A, B, C, F, G, H)$ are arbitrary coefficients; if they are the coordinates of a line, that is, if $A F+B G+C H=0$, then the condition expresses that the line $(a, b, c, f, g, h)$, instead of belonging to a proper involution, meets the line $(F, G, H, A, B, C)$.

1 The theory is explained in my memoir "On the Six Coordinates of a Line," Camb. Phil. Trans. vol. xI. 1868, [348]. In explanation of the subsequent analytical investigations of the present memoir, it is convenient to remark that if on a given line we have the two points $(a, \beta, \gamma, \delta)$ and $\left(\alpha^{\prime}, \beta^{\prime}, \gamma^{\prime}, \delta^{\prime}\right)$, and through the given line two planes $A x+B y+C z+D w=0$ and $A^{\prime} x+B^{\prime} y+C^{\prime} z+D^{\prime} w=0$; then we have

$$
\begin{aligned}
\beta \gamma^{\prime}-\beta^{\prime} \gamma: \gamma \alpha^{\prime}-\gamma^{\prime} a: a \beta^{\prime}-a^{\prime} \beta: a \delta^{\prime}-a^{\prime} \delta: \beta \delta^{\prime}-\beta^{\prime} \delta: \gamma \delta^{\prime}-\gamma^{\prime} \delta \\
=A D^{\prime}-A^{\prime} D: B D^{\prime}-B^{\prime} D: C D^{\prime}-C^{\prime} D: B C^{\prime}-B^{\prime} C: C A^{\prime}-C^{\prime} A: A B^{\prime}-A^{\prime} B ;
\end{aligned}
$$

and denoting either of these sets of equal ratios by

then $(a, b, c, f, g, h)$ satisfy identically the relation $a f+b g+c h=0$, and are said to be the six coordinates of the line. 
We have to determine the locus of the line $(a, b, c, f, g, h)$ the coordinates whereof satisfy the relation

$$
(A, B, C, F, G, H \gamma a, b, c, f, g, h)=0,
$$

and which besides meets the skew cubic $y w-z^{2}=0, y z-x w=0, x z-y^{2}=0$.

The equations of the skew cubic are satisfied by writing therein

$$
x: y: z: w=1: t: t^{2}: t^{3} ;
$$

and hence taking $\theta, \phi$ for the parameters of the points of intersection of the line $(a, b, c, f, g, h)$ with the skew cubic, we have

$$
\begin{array}{llll}
1, & \theta, & \theta^{2}, & \theta^{3} \\
1, & \phi, & \phi^{2}, & \phi^{3},
\end{array}
$$

as the coordinates of two points on the line in question; whence forming the expressions of the six coordinates of the line, and omitting the common factor $\phi-\theta$, these are

$$
(a, b, c, f, g, h)=\theta \phi,-(\theta+\phi), 1, \theta^{2}+\theta \phi+\phi^{2}, \theta \phi(\theta+\phi), \theta^{2} \phi^{2},
$$

and hence the condition of invoi ion gives between the parameters $\theta, \phi$ the equation

$$
\left(A, B, C, F, G, H \gamma \theta \phi,-\theta-\phi, 1, \theta^{2}+\theta \phi+\phi^{2}, \theta \phi(\theta+\phi), \theta^{2} \phi^{2}\right) .
$$

Moreover the coordinates of any point on the line in question are given by

$$
x: y: z: w=l+m: l \theta+m \phi: l \theta^{2}+m \phi^{2}: l \theta^{3}+m \phi^{3}
$$

and writing as above $p, q, r=y w-z^{2}, y z-x w, x z-y^{2}$, we thence find, omitting the common factor $(\theta-\phi)^{2}$,

$$
p: q: r=\theta \phi:-(\theta+\phi): 1
$$

and eliminating $\theta \phi, \theta+\phi$, we at once obtain

$$
\left(A, B, C, F, G, H \chi p r, q r, r^{2}, q^{2}-p r,-p q, p^{2}\right)=0,
$$

or, what is the same thing,

$$
\left(H, F, C, B, A-F,-G(p, q, r)^{2}=0\right.
$$

as the equation of the scroll generated by the line in involution which meets the given skew cubic twice.

\section{Reciprocal of the Quartic Scroll $S\left(3^{2}\right)$.}

67. I propose to reciprocate in regard to the quadric surface $x^{2}+y^{2}+z^{2}+t^{2}=0$ the foregoing scroll

$$
\left(H, F, C, B, A-F,-G^{\gamma}(p, q, r)^{2}=0 .\right.
$$

If the coordinates $(a, b, c, f, g, h)$ of a line satisfy the condition of involution

$$
(A, B, C, F, G, H \nmid a, b, c, f, g, h)=0 \text {, }
$$


then the coordinates $(a, b, c, f, g, h)$ of the reciprocal curve will satisfy the condition of involution

$$
(F, G, H, A, B, C \gamma a, b, c, f, g, h)=0 .
$$

The reciprocal of the before-mentioned skew cubic $x: y: z: w=1: t: t^{2}: t^{3}$ is the quartic torse having for its edge of regression the skew cubic $3 X Z-Y^{2}=0, Y Z-9 X W=0$, $3 Y W-Z^{2}=0$; or, what is the same thing, the skew cubic $X: Y: Z: W=1: 3 t: 3 t^{2}: t^{3}$; see my paper "On the Reciprocation of a Quartic Developable," Quart. Math. Journ. vol. vII. (1866), pp. 87-92, [372].

68. Hence the reciprocal of the quartic scroll is the scroll generated by a line $(a, b, c, f, g, h)$ the coordinates of which satisfy the condition of involution

$$
(F, G, H, A, B, C \gamma(a, b, c, f, g, h)=0,
$$

and which is moreover the intersection of two osculating planes of the skew cubic $X: Y: Z: W=1: 3 t: 3 t^{2}: t^{3}$. For the point the parameter whereof is $t$, the equation of the osculating plane is

$$
\left|\begin{array}{cccc}
X, & Y, & Z, & W \\
1, & 3 t, & 3 t^{2}, & t^{3} \\
1, & 2 t, & t^{2} \\
& 1, & t
\end{array}\right|=0
$$

or, what is the same thing, the equation is

$$
\left(t^{3},-t^{2}, t,-1 \gamma X, Y, Z, W\right)=0 .
$$

Hence for the line which is the intersection of the two osculating planes

$$
\begin{aligned}
& \left(\theta^{3},-\theta^{2}, \theta,-1 \gamma X, Y, Z, W\right)=0, \\
& \left(\phi^{3},-\phi^{2}, \phi,-1 \gamma X, Y, Z, W\right)=0,
\end{aligned}
$$

forming the expressions of the six coordinates, but omitting the common factor $\phi-\theta$, these are

$$
a, b, c, f, g, h=\theta^{2}+\theta \phi+\phi^{2},-\theta-\phi, 1, \theta \phi, \theta \phi(\theta+\phi), \theta^{2} \phi^{2}
$$

we have thus between the parameters $\theta, \phi$ the relation

$$
\left(F, G, H, A, B, C \gamma \theta^{2}+\theta \phi+\phi^{2},-\theta-\phi, 1, \theta \phi, \theta \phi(\theta+\phi), \theta^{2} \phi^{2}\right)=0 ;
$$

and the equation of the scroll is obtained by eliminating $\theta, \phi$ between this equation and the last-mentioned two equations satisfied by $\theta, \phi$ respectively.

69. We see that $\theta, \phi$ are two of the roots of the equation

$$
\left(X,-Y, Z,-W^{\gamma}(u, 1)^{3}=0 ;\right.
$$


let $\rho$ be the third root, then we have

and thence

$$
\begin{array}{ll}
\theta+\phi+\rho & =\frac{Y}{X}, \\
\theta \phi+\rho(\theta+\phi) & =\frac{Z}{X}, \\
\theta \phi \cdot \rho & =\frac{W}{X},
\end{array}
$$

$$
\begin{gathered}
\theta+\phi=\frac{1}{X}(Y-\rho X), \quad \theta \phi=\frac{1}{X}\left(Z-\rho Y+\rho^{2} X\right)=\frac{1}{\rho X} W, \\
\left(X,-Y, Z,-W \gamma(\rho, 1)^{3}=0 .\right.
\end{gathered}
$$

Substituting for $\theta+\phi$ and $\theta \phi$ their values in terms of $\rho$, we find

$$
\begin{aligned}
F \rho\left\{Y^{2}-Z X-\rho X Y\right\}-G \rho X(Y-\rho X)+H \rho X^{2} \\
+W\left\{(A X+B Y+C Z)-\rho(B X+C Y)+\rho^{2} C X\right\}=0,
\end{aligned}
$$

or, what is the same thing,

$$
\begin{aligned}
& \rho^{2} X(G X-F Y+C W) \\
- & \rho\left\{F\left(Y^{2}-Z X\right)-G X Y+H X^{2}-B X W-C Y W\right\} \\
+ & W(A X+B Y+C Z)=0
\end{aligned}
$$

from which and the equation

$$
\left(X,-Y, Z,-W^{\gamma}(\rho, 1)^{3}=0,\right.
$$

we have to eliminate $\rho$.

70. Writing for shortness

$$
\begin{array}{rlrl}
(, H,-G, A \gamma X, Y, Z, W) & =\alpha, \\
(-H, ., F, B \gamma & & =\beta, \\
(G,-F, ., C \gamma & & =\gamma, \\
(-A,-B,-C, . \gamma & & & =\delta,
\end{array}
$$

and therefore $\alpha X+\beta Y+\gamma Z+\delta W=0$ : the two equations are

$$
\begin{aligned}
\dot{\rho}^{2} X \gamma+\rho(-\gamma Y-\beta X)-\delta W & =0, \\
\rho^{3} X-\rho^{2} Y+\rho Z & -W=0 .
\end{aligned}
$$

Writing the first equation in the form

$$
\gamma\left(\rho^{2} X-\rho Y+Z\right)-\beta(\rho X-Y)+\alpha X=0,
$$

multiplying by $-\rho$, and reducing by the other equation,

$$
\beta\left(\rho^{2} X-\rho Y\right)-\rho \alpha X-\gamma W=0,
$$

or, as this may be written,

$$
\beta\left(\rho^{2} X-\rho Y+Z\right)-\alpha(\rho X-Y)-\alpha Y-\beta Z-\gamma W=0 .
$$


From this and the preceding equation we deduce the values of $\rho^{2} X-\rho Y+Z$ and $\rho X-Y$; viz. writing for shortness

we find

$$
\beta \delta-\gamma^{2}, \beta \gamma-\alpha \delta, \alpha \gamma-\beta^{2}=p, q, r,
$$

$$
\rho^{2} X-\rho Y+Z: \rho X-Y: 1=-\mathrm{r} Z+\mathrm{q} W: \mathrm{r} Y-\mathrm{p} W:-\mathrm{r},
$$

or, what is the same thing,

whence also

$$
\begin{array}{ll}
\rho^{2} X-\rho Y+Z & =Z-\frac{\mathrm{q}}{\mathrm{r}} W \\
\rho X-Y & =-Y+\frac{\mathrm{p}}{\mathrm{r}} W
\end{array}
$$

$$
\begin{array}{ll}
\rho^{3} X-\rho^{2} Y+\rho Z-W & =0, \\
\rho^{2} X-\rho Y & =-\frac{q}{r} W, \\
\rho X & =\frac{p}{r} W,
\end{array}
$$

and thence

$$
\begin{array}{ll}
\rho\left(Z-\frac{\mathrm{q}}{\mathrm{r}} W\right) & =W, \\
\rho\left(Y-\frac{\mathrm{p}}{\mathrm{r}} W\right) & =\frac{\mathrm{q}}{\mathrm{r}} W, \\
\rho X & =\frac{\mathrm{p}}{\mathrm{r}} W,
\end{array}
$$

and we have therefore

$$
\left(\frac{W}{\rho}=\right) \quad Z-\frac{\mathrm{q}}{\mathrm{r}} W=\frac{\mathrm{r}}{\mathrm{q}}\left(Y-\frac{\mathrm{p}}{\mathrm{r}} W\right)=\frac{\mathrm{r}}{\mathrm{p}} X,
$$

or omitting the first equation, we have (independent of $\rho$ ) a system which it is clear must be equivalent to a single equation.

71. I take any one of these equations, for instance the equation

or, what is the same thing,

$$
Z-\frac{\mathrm{q}}{\mathrm{r}} W=\frac{\mathrm{r}}{\mathrm{q}}\left(Y-\frac{\mathrm{p}}{\mathrm{r}} W\right),
$$

$$
\mathrm{qr} Z-\mathrm{r}^{2} Y+\left(\mathrm{pr}-\mathrm{q}^{2}\right) W=0,
$$

and I proceed to reduce it so as to obtain the result in a symmetrical form. For this purpose I observe that from the values of $\alpha, \beta, \gamma, \delta$, if only $A F+B G+C H$ not $=0$, we have

$$
\begin{aligned}
& X: Y: Z: W=(.,-C, \quad B,-F \gamma(\alpha, \beta, \gamma, \delta) \\
& :(C, \quad,-A,-G \gamma \quad,) \\
& :(-B, \quad A, \quad \ldots,-H \chi \quad, \quad)
\end{aligned}
$$

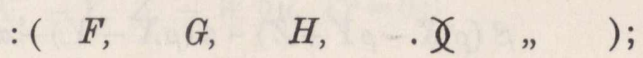


and substituting these values, the equation in question becomes

This becomes

$$
\begin{gathered}
\mathrm{qr} \quad(-B \alpha+A \beta-H \delta) \\
-\mathrm{r}^{2}(C \alpha-A \gamma-G \delta) \\
+\left(\mathrm{pr}-\mathrm{q}^{2}\right)(\quad F \alpha+G \beta+H \gamma)=0 .
\end{gathered}
$$

$$
\begin{aligned}
& A \mathrm{r}(\mathrm{q} \beta+\mathrm{r} \gamma) \\
& =A \mathrm{r}(-\mathrm{p} \alpha)=0 \\
& \text { - Bqra } \\
& -B q r a \\
& -\mathrm{Cr}^{2} \alpha \\
& -C \mathrm{r}^{2} \alpha \\
& +F\left(\mathrm{pr}-\mathrm{q}^{2}\right) \alpha \\
& +F\left(\mathrm{pr}-\mathrm{q}^{2}\right) \alpha \\
& +G\left\{\mathrm{r}^{2} \delta+\left(\mathrm{pr}-\mathrm{q}^{2}\right) \beta\right\} \\
& +G \mathrm{pq} \alpha \\
& +H\left\{-\mathrm{qr} \delta+\left(\mathrm{pr}-\mathrm{q}^{2}\right) \gamma\right\} \\
& -H \mathrm{p}^{2} \alpha \text {, }
\end{aligned}
$$

viz. the whole equation divides by $\alpha$; and, omitting this factor, the equation is

$$
A \mathrm{pr}+B \mathrm{qr}+C \mathrm{r}^{2}+F\left(\mathrm{q}^{2}-\mathrm{pr}\right)-G \mathrm{pq}+H \mathrm{p}^{2}=0,
$$

or, what is the same thing, it is

$$
\left(H, F, C, B, A-F,-G^{\gamma}(\mathrm{p}, \mathrm{q}, \mathrm{r})^{2}=0,\right.
$$

where I recall that we have

$$
\mathrm{p}, \mathrm{q}, \mathrm{r}=\beta \delta-\gamma^{2}, \beta \gamma-\alpha \delta, \alpha \gamma-\beta^{2},
$$

$\alpha, \beta, \gamma, \delta$ being linear functions of the current coordinates $(X, Y, Z, W)$, viz. we have

$$
\begin{aligned}
& \alpha=(., \quad H,-G, A \gamma X, Y, Z, W) \text {, } \\
& \beta=(-H, \quad \text {, } \quad F, \quad B \gamma \quad, \quad) \text {, }
\end{aligned}
$$

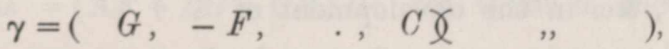

$$
\begin{aligned}
& \delta=(-A,-B,-C, \cdot \gamma \quad, \quad) \text {. }
\end{aligned}
$$
scroll

72. It thus appears that when $A F+B G+C H$ is not $=0$, the reciprocal of the

$$
\left(H, F, C, B, A-F,-G^{r}(p, q, r)^{2}=0\right.
$$

has an equation of the very same form,

$$
\left(H, F, C, B, A-F,-G^{\gamma}(\mathrm{p}, \mathrm{q}, \mathrm{r})^{2}=0 ;\right.
$$

so that in fact the scroll, tenth species, $S\left(3^{2}\right)$, defined as the scroll generated by a line in involution which passes through two points of a skew cubic, may be reciprocally defined as the scroll generated by a line in invoiution which lies in two osculating planes of a skew cubic.

73. If for $(\alpha, \beta, \gamma, \delta)$ we substitute their values in terms of $(X, Y, Z, W)$, the foregoing equation of the reciprocal scroll is obtained as an equation of the fourth order in the coordinates $(X, Y, Z, W)$, and (in the first instance) of the fifth degree in the coefficients $(A, B, C, F, G, H)$. It is a remarkable circumstance that the whole c. VI. 
equation contains the constant factor $A F+B G+C H$, so that throwing this out, the reduced equation will be only of the third degree in the coefficients.

74. The transformation is a very troublesome one, but I will indicate the steps by which I succeeded in accomplishing it. Each of the functions (p, q, r) is a quadric function of $(X, Y, Z, W)$, say,

$$
\begin{aligned}
& \mathrm{p}=\left(a, b, c, d, f, g, h, l, m, n^{\gamma} X, Y, Z, W\right)^{2}, \\
& q=\left(a^{\prime}, \ldots \quad \gamma \quad\right. \text {. } \\
& \mathrm{r}=\left(a^{\prime \prime}, \ldots \quad \gamma \quad \text { " } \quad\right)^{2} \text {; }
\end{aligned}
$$

we have to form the value of

$$
(H, F, C, B, A-F,-G \gamma \mathrm{\gamma}, \mathrm{q}, \mathrm{r})^{2},
$$

viz. representing this for shortness by

$$
(H, F, C, B, A-F,-G)\left(\begin{array}{l}
a, b, c, d, f, g, h, l, m, n \\
a^{\prime}, . . \\
a^{\prime \prime}, . .
\end{array}\right)^{2}
$$

the coefficient of $X^{4}$ is

$$
\left(H, F, C, B, A-F,-G^{r}\left(a^{2}, a^{\prime 2}, a^{\prime \prime 2}, a^{\prime} a^{\prime \prime}, a^{\prime \prime} a, a a^{\prime \prime}\right),\right.
$$

that of $X^{3} Y$ is

$$
\left(H, F, C, B, A-F,-G \gamma 2 a f, 2 a^{\prime} f^{\prime}, 2 a^{\prime \prime} f^{\prime \prime}, a^{\prime} f^{\prime \prime}+a^{\prime \prime} f^{\prime}, a^{\prime \prime} f+a f^{\prime \prime}, a f^{\prime}+a^{\prime} f\right),
$$

and so on, the successive terms $a^{2}, a^{\prime 2}$, \&c., $2 a f, 2 a^{\prime} f^{\prime}$, \&c. being derived by an obvious law from the first terms $a^{2}, 2 a f$, \&c.; and these first terms are merely the coefficients of the terms $X^{4}, X^{3}, Y, \& c$. in the development of

viz. this is

$$
\left.p^{2},=\{(a, b, c, d, f, g, h, l, m, n\} X, Y, Z, W)^{2}\right\}^{2} ;
$$

$X^{4} X^{3} Y X^{3} Z X^{3} W X^{2} Y^{2} X^{2} Y Z X^{2} Y W X^{2} Z^{2}, X^{2} Z W, X^{2} W^{2}, X Y^{3}, X Y^{2} Z, X Y^{2} W, X Y Z^{2}$

\begin{tabular}{lllllllllllllr}
\hline$a^{2}$ & $2 a f$ & $2 a g$ & $2 a l$ & $2 a b$ & $2 a f$ & $2 a m$ & $2 a c$ & $2 a n$ & $2 a d$ & $2 b h$ & $2 b g$ & $2 b l$ & $2 c h$ \\
& & $+h^{2}$ & $+2 g h$ & $+2 h l$ & $+g^{2}$ & $+2 g l$ & $+l^{2}$ & & $+2 f h$ & $+2 h m$ & $+2 f g$
\end{tabular}

$X Y Z W, X Y W^{2}, X Z^{3}, X Z^{2} W, X Z W^{2}, X W^{3}, Y^{4}, Y^{3} Z, Y^{3} W, Y^{2} Z^{2}, Y^{2} Z W, Y^{2} W^{2}, Y Z^{3}$,

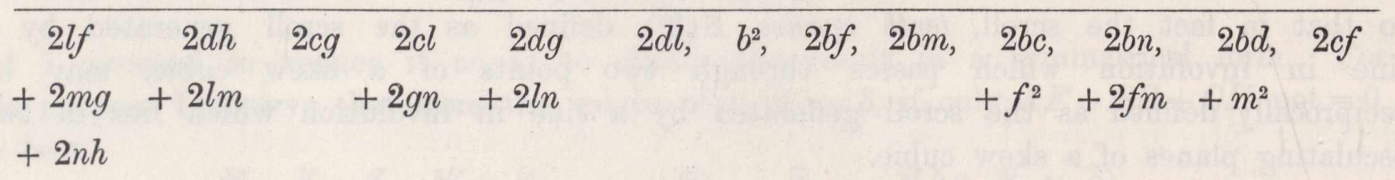

$Y Z^{2} W, Y Z W^{2}, Y W^{3}, Z^{4}, Z^{3} W, Z^{2} W^{2}, Z W^{3}, W^{4}$

$2 c m+2 d f \quad 2 d m, c^{2}, 2 c n, \quad 2 c d, 2 d n, d^{2}$
$+2 f n+2 m n$
$+n^{2}$


and the values of the coefficients $a, b, \ldots$ which enter into the formulæ are given by means of the following values of $p, q, r$; viz. these are

$$
\begin{aligned}
& \begin{array}{llllllllll}
X^{2} & Y^{2} & Z^{2} & W^{2} & Y Z & Z X & X Y & X W & Y W & Z W
\end{array} \\
& \mathrm{p}=\left(\begin{array}{r}
\left.A H,-F^{2},-C F,-C^{2},-B F,-A F, \quad B H,-A B,-B^{2},-B C_{\gamma}^{\gamma} X, Y, Z, W\right)^{2}, \\
-G^{2}, C H+2 F G-2 C G+2 C F
\end{array}\right.
\end{aligned}
$$

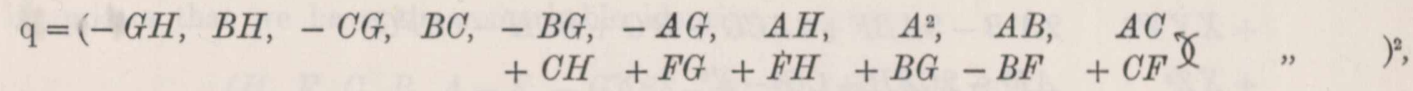

$$
\begin{aligned}
& -F^{2}-C H
\end{aligned}
$$

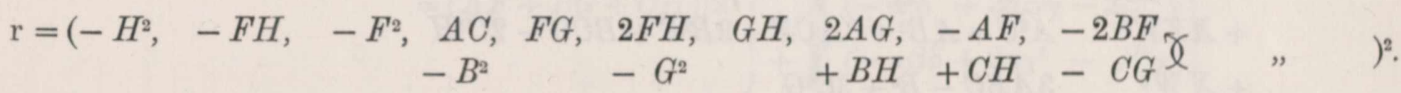

75. As an instance of the calculation of a single term, the coefficient of $X^{4}$ is

$$
\left(H, F, C, B, A-F,-G \gamma A H-G^{2},-G H,-H^{2}\right)^{2} ;
$$

viz. this is

$$
\begin{array}{rlr}
H\left(A H-G^{2}\right)^{2} & = & A^{2} H^{3}-2 A G^{2} H^{2}+G^{4} H^{2} \\
+F H^{2} G^{2} & = & F G^{2} H^{2} \\
+C H^{4} & = & C H^{4} \\
+B G H^{3} & =B G H^{3} \\
+\left(A-F^{2}\right)\left(-A H^{3}+G^{2} H^{2}\right)= & -A^{2} H^{3}+A G^{2} H^{2} \\
& +A F H^{3}-F G^{2} H^{2} \\
-G\left(-A G H^{2}+G^{3} H\right) & =A G^{2} H^{2}-G^{4} H^{2}
\end{array}
$$

the whole term is thus $=(A F+B G+C H) H^{3}$, viz. there is the factor $A F+B G+C H$ as mentioned above.

76. Throwing out the factor in question, $A F+B G+C H$, the equation of the reciprocal scroll is found to be

$$
\begin{aligned}
& 0=X^{4} \cdot H^{3} \\
& +X^{3} Y \quad-2 G H^{2} \\
& +X^{3} Z \quad \cdot A H^{2}-3 F H^{2}+G^{2} H \\
& +X^{3} W \quad-3 A G H-3 B H^{2}+G^{3} \\
& +X^{2} Y^{2} \quad \cdot 2 F^{2}+G^{2} H \\
& +X^{2} Y Z .-A G H+B H^{2}+2 F G H-G^{3} \\
& +X^{2} Y W . \quad A^{2} H+3 A F H+A G^{2}+B G H-2 C H^{2}-3 F G^{2} \\
& +X^{2} Z^{2} \quad .-2 A F H+A G^{2}+C H^{2}-F G^{2}+3 F^{2} H \\
& +X^{2} Z W .-2 A^{2} G-2 A B H+3 A F G+6 B F H-B G^{2}-C G H \\
& +\mathrm{X}^{2} W^{2} . A^{3}+3 A B G-3 A C H+3 B^{2} H+3 C G^{2}
\end{aligned}
$$




$$
\begin{aligned}
& +X Y^{3} \quad-2 F G H \\
& +X Y^{2} Z \quad A F H-B G H-3 F^{2} H+2 F G^{2} \\
& +X Y^{2} W \quad 2 A B H-2 A F G-B F H+2 C G H+3 F^{2} G \\
& +X Y Z^{2}-A F G-2 B F H+B G^{2}-C G H \\
& +X Y Z W \quad A^{2} F^{\prime}-3 A F^{2}-3 A B G+A C H-2 B^{2} H+B F G+5 C F H-2 C G^{2} \\
& +X Y W^{2} \quad 2 A^{2} B-3 A B F+2 A C G+B^{2} G+B C H-6 C F G \\
& +X Z^{3} \quad A F^{2}-2 C F H+C G^{2}-F^{3} \\
& +X Z^{2} W \quad 2 A B F-2 A C G-2 B C H-3 B F^{2}+C F G \\
& +X Z W^{2} \quad A^{2} C+A B^{2}+3 A C F-3 B^{2} F+B C G-2 C^{2} H \\
& +X W^{3} \quad 3 A B C-B^{3}+3 C^{2} G \\
& +Y^{4} \quad F^{2} H \\
& +Y^{3} Z \quad B F H-F^{2} G \\
& +Y^{3} W \quad A F^{2}+B^{2} H-2 C F H-F^{3} \\
& +Y^{2} Z^{2}-B F G+C F H+F^{3} \\
& +Y^{2} Z W \quad A B F-B^{2} G+B C H+2 C F G \\
& +Y^{2} W^{2} \quad A B^{2}-2 A C F-B^{2} F+C^{2} H+3 C F^{2} \\
& +Y^{3} \quad B F^{2}-C F G \\
& +Y Z^{2} W \quad A C F+2 B^{2} F-B C G-3 C F^{2} \\
& +Y Z W^{2} \quad A B C+B^{3}-2 B C F-\mathcal{C}^{2} G \\
& +Y W^{3} \quad A C^{2}+B^{2} C-3 C^{2} F \\
& +Z^{4} \quad C F^{2} \\
& +Z^{3} W \quad 2 B C F \\
& +Z^{2} W^{2} \quad B^{2} C+2 C^{2} F^{\prime} \\
& +Z W^{3} \quad 2 B C^{2} \\
& +W^{4} \quad C^{3} \text {, }
\end{aligned}
$$

where, in regard to the symmetry of this equation, it is to be observed that we may interchange $X$ and $W$, and $Y$ and $Z$, leaving $A, F$ unaltered but interchanging $B$ and $-G$, and also $C$ and $H$; thus the coefficient of $X^{3} Z$ being $A H^{2}-3 F H^{2}+G^{2} H$, that of $Y W^{3}$ is $A C^{2}-3 F C^{2}+B^{2} C,=A C^{2}+B^{2} C-3 C^{2} F$. Or, again, the coefficient of $Y^{3} Z$ being $B F^{\prime} H-F^{2} G$, that of $Y Z^{3}$ is $-G F C+F^{2} B,=B F^{2}-C F G$.

77. But the equation may be written in the much more simple form

$$
\begin{aligned}
& X\left(-\alpha^{2} \delta+3 \alpha \beta \gamma-2 \beta^{3}\right) \\
+ & Y\left(-\alpha \beta \delta+2 \alpha \gamma^{2}-\beta^{2} \gamma\right) \\
+ & Z\left(a \gamma \delta-2 \beta^{2} \delta+\beta \gamma^{2}\right) \\
+ & W\left(a \delta^{2}-3 \beta \gamma \delta+2 \gamma^{3}\right)=0,
\end{aligned}
$$


or, what is the same thing,

$$
-\frac{1}{6}\left(3 X \partial_{\delta}-Y \partial_{\gamma}+Z \partial_{\beta}-3 W \partial_{\alpha}\right)\left(\alpha^{2} \delta^{2}-6 \alpha \beta \gamma \delta+4 \alpha \gamma^{3}+4 \beta^{3} \delta-3 \beta^{2} \gamma^{2}\right)=0
$$

as may be verified by actual substitution of the values of the coordinates.

78. By what precedes, substituting for $\mathrm{p}, \mathrm{q}, \mathrm{r}$ their values in terms of $\alpha, \beta, \gamma, \delta$, it appears that we have the remarkable identity

$$
\begin{aligned}
& \left(H, F, C, B, A-F,-G \gamma \beta \beta \delta-\gamma^{2}, \beta \gamma-\alpha \delta, \alpha \gamma-\beta^{2}\right)^{2} \\
& =(A F+B G+C H) \times\left\{\begin{array}{c}
X\left(-\alpha^{2} \delta+3 \alpha \beta \gamma-2 \beta^{3}\right) \\
+Y\left(-\alpha \beta \delta+2 \alpha \gamma^{2}-\beta^{2} \gamma\right) \\
+Z\left(a \gamma \delta-2 \beta^{2} \delta+\beta \gamma^{2}\right) \\
+W\left(a \delta^{2}-3 \beta \gamma \delta+2 \gamma^{3}\right)
\end{array}\right\} .
\end{aligned}
$$

79. In the case above considered of the tenth species, $S\left(3^{2}\right)$, for which $A F+B G+C H$ not $=0$, the three forms of the reciprocal equation are of course absolutely equivalent to each other. The first form has the advantage of putting in evidence the fact that the reciprocal scroll is also of the tenth species; the other two forms do not, at least obviously, put in evidence any special property of the reciprocal scroll.

Reciprocals of Eighth Species, $S\left(1,3^{2}\right)$, and Ninth Species, $S\left(1^{3}\right)$.

80. If $A F+B G+C H=0$, then the equation

$$
\left(H, F, C, B, A-F,-G^{r}(p, q, r)^{2}=0\right.
$$

is a scroll of the eighth species, $S\left(1,3^{2}\right)$. The first form of the reciprocal equation becomes identically $0=0$, on account of the evanescent factor $A F+B G+C H$, but the second and third forms continue to subsist, and either of them may be taken as the equation of the reciprocal scroll. Taking the third form, and calling to mind the significations of $(\alpha, \beta, \gamma, \delta)$, viz.

$$
\begin{aligned}
& \alpha=(., \quad H,-G, A \gamma X, Y, Z, W) \text {, } \\
& \beta=(-H, \quad ., \quad F, B \gamma \quad \text { " }) \text {, } \\
& \gamma=(G,-F, \quad, C \gamma \quad, \quad) \text {, } \\
& \delta=(-A,-B,-C, \gamma \quad, \quad),
\end{aligned}
$$

it is to be observed that $\alpha=0, \beta=0, \gamma=0, \delta=0$ are the equations of four planes passing through a common line, viz. the line whose coordinates are $(A, B, C, F, G, H)$, and the equation thus puts in evidence that this line is a triple line on the reciprocal 
scroll; that is, the reciprocal scroll is a scroll of the ninth species, $S\left(1^{3}\right)$. Or stating the theorem more completely: For the scroll, eighth species, $S\left(1,3^{2}\right)$,

$$
\left(H, F, C, B, A-F,-G^{\gamma}(\mathrm{p}, \mathrm{q}, \mathrm{r})^{2}=0,\right.
$$

generated by a line meeting the line $(F, G, H, A, B, C)$, and the skew cubic $\mathrm{p}=0$ $\mathrm{q}=0, \mathrm{r}=0$ twice, the reciprocal scroll is of the ninth species, $S\left(1^{3}\right)$,

$$
\begin{aligned}
& X\left(-\alpha^{2} \delta+3 \alpha \beta \gamma-2 \beta^{3}\right) \\
+ & Y\left(-\alpha \beta \delta+2 \alpha \gamma^{2}-\beta^{2} \gamma\right) \\
+ & Z\left(\alpha \gamma \delta-2 \beta^{2} \delta+\beta \gamma^{2}\right) \\
+ & W\left(\alpha \delta^{2}-3 \beta \gamma \delta+2 \gamma^{3}\right)
\end{aligned}
$$

having for its triple line the reciprocal line $(A, B, C, F, G, H)$.

81. It should of course be possible, starting from the equation

$$
(* \gamma X, Y)^{4}+Z(* \chi X, Y)^{3}+W\left(*^{\prime} \gamma X, Y\right)^{3}=0
$$

of a scroll $S\left(1^{3}\right)$, to obtain the equation of the reciprocal scroll $S\left(1,3^{2}\right)$. But I content myself with a very particular case. I consider the equation

$$
Y^{2} Z^{2}-Y^{3} W-Z^{3} X=0,
$$

which belongs to a scroll $S\left(1^{3}\right)$ having the line $Y=0, Z=0$ for its triple line. To find the equation of the reciprocal scroll, write

$$
\begin{array}{r}
-Z^{3}+\lambda x=0 \\
2 Y Z^{2}-3 Y^{2} W+\lambda y=0 \\
2 Y^{2} Z-3 Z^{2} X+\lambda z=0 \\
-Y^{3}+\lambda w=0
\end{array}
$$

we find without difficulty, reducing by means of the equation of the scroll,

$$
\begin{aligned}
& \lambda^{2}\left(y w-z^{2}\right)=-3 Z^{2}\left\{Y^{4}+3 X Z\left(X Z-Y^{2}\right)\right\} \\
& \lambda^{2}(x w-y z)=3 Y^{2} Z^{2}\{Y Z-3 W X\} \\
& \lambda^{2}\left(x z-y^{2}\right)=-3 Y^{2}\left\{Z^{4}+3 Y W\left(Y W-Z^{2}\right)\right\} .
\end{aligned}
$$

Hence writing for a moment

$$
\Omega=\left\{Y^{4}+3 X Z\left(X Z-Y^{2}\right)\right\}\left\{Z^{4}+3 Y W\left(Y W-Z^{2}\right)\right\}-Y^{2} Z^{2}(Y Z-3 W X)^{2},
$$

we have

$$
\begin{aligned}
\Omega= & Y^{4} Z^{4}+3 Y^{5} W\left(Y W-Z^{2}\right)+3 Z^{5} X\left(X Z-Y^{2}\right)+9 X Y Z W\left(Y^{2} Z^{2}-Y^{3} W-Z^{3} X+X Y Z W\right) \\
& -Y^{4} Z^{4}+6 Y^{3} Z^{3} X W-9 Y^{2} Z^{2} X^{2} W^{2},
\end{aligned}
$$


that is

$$
\begin{aligned}
\frac{1}{3} \Omega & =Y^{5} W\left(Y W-Z^{2}\right)+X^{5} X\left(X Z-Y^{2}\right)+2 Y^{3} Z^{3} X W, \\
& =-Y^{2} Z^{2}\left(X Z-Y^{2}\right)\left(Y W-Z^{2}\right)-Y^{2} Z^{2}\left(Y W-Z^{2}\right)\left(X Z-Y^{2}\right)+2 Y^{3} Z^{3} X W, \\
& =-2 Y^{2} Z^{2}\left\{\left(X Z-Y^{2}\right)\left(Y W-Z^{2}\right)-X Y Z W\right\}, \\
& =-2 Y^{2} Z^{2}\left\{Y^{2} Z^{2}-Y^{3} W-Z^{3} X\right\}, \\
& =0, \text { by the equation of the scroll; }
\end{aligned}
$$

and we thus see that the equation of the reciprocal scroll is

$$
\left(y w-z^{2}\right)\left(x z-y^{2}\right)-(y z-x w)^{2}=0,
$$

or say $\mathrm{q}^{2}-\mathrm{pr}=0$, viz. it is a scroll $S\left(1,3^{2}\right)$ generated by a line meeting the line $x=0, w=0$, and the cubic curve $\mathrm{p}=0, \mathrm{q}=0, \mathrm{r}=0$ twice. The equation is obviously included in the general equation

$$
\left(H, F, C, B, A-F,-G^{r} \mathrm{\gamma}, \mathrm{q}, \mathrm{r}\right)^{2}=0,
$$

where $A F+B G+C H=0$; viz. writing $A=B=C=G=H=0$, this becomes $F\left(q^{2}-p r\right)=0$.

82. Returning to the general case of the scroll, eighth species, $S\left(1,3^{2}\right)$, it is proper to show geometrically how it is that the reciprocal is a scroll, ninth species, $S\left(1^{3}\right)$. Consider in the scroll $S\left(1,3^{2}\right)$ any plane through the directrix line; this contains three generating lines of the scroll, viz. these are the sides of the triangle formed by the three points of intersection of the plane with the skew cubic: hence in the reciprocal figure we have a directrix line such that at each point of it there are three generating lines; that is, we have a scroll $S\left(1^{3}\right)$ with a triple directrix line. Conversely, starting with the scroll $S\left(1^{3}\right)$, each plane through the triple directrix line meets the scroll in this line three times, and in a single generating line; whence there is in the reciprocal scroll a simple directrix line; but in order to show that it is a scroll $S\left(1,3^{2}\right)$, we have yet to show that there is, as a nodal directrix, a skew cubic met twice by each generating line; this implies that, reciprocally, in the scroll $S\left(1^{3}\right)$ each generating line is the intersection of two osculating planes of a skew cubic (tangent planes of a quartic torse), each such plane containing two generating lines of the scroll-a geometrical property which is far from obvious; and similarly in the scroll, ninth species, $S\left(3^{2}\right)$, where the reciprocal scroll is of the same form, the property that each generating line is a line joining two points of a skew cubic leads to the property that each line is also the intersection of two osculating planes of a skew cubic (or, what is the same thing, two tangent planes of a quartic torse).

AdDition, May 18, 1869.

Since the foregoing Memoir was written I received from Professor Cremona a letter dated Milan, November 20, 1868, in which (besides the ninth and tenth species considered above) he refers to two other species of quartic scrolls. He remarks that 
there is a bitangent torse which should in the classification be considered along with the nodal curve; and he enumerates in all 12 species as follows:

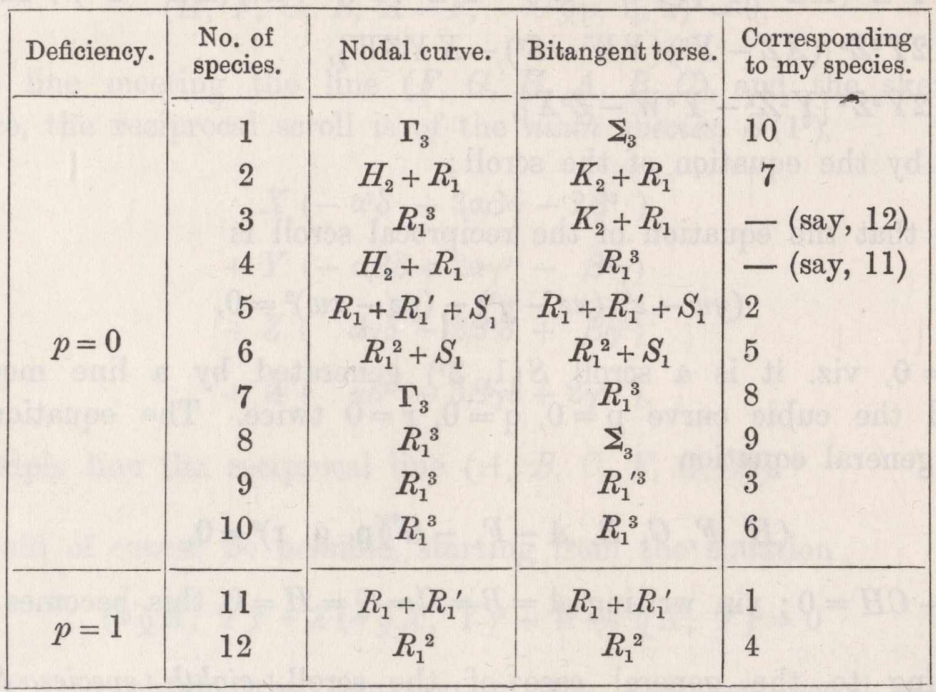

where $\Gamma_{3}$ denotes a skew cubic, $\Sigma_{3}$ a torse of the 3rd class (or quartic torse), $H_{2}$ a conic, $K_{2}$ a quadric cone, $R_{1}, R_{1}^{\prime}, S_{1}$ different right lines, $R_{1}^{2}, R_{1}^{3}$ a line counted twice or three times, \&c. I have in the last column added the references to my species 9 and 10; Professor Cremona notices 'what I knew, but did not recollect) that the species 10 had been considered by M. Chasles, Comptes Rendus, June 3, 1861.

I have not yet examined the two new species mentioned in this enumeration; viz. these are (Cremona 3), say twelfth species, a scroll having a triple line, but a bitangent torse made up of a quadric cone and a line; and (Cremona 4), say eleventh species, a scroll having a nodal conic and line, but for its bitangent torse a triple line: the two species are, it is clear, reciprocal to each other; although properly treated as distinct, species 11 may be considered as a subform of 8 , and species 12 as a subform of 9 . 